\title{
On the unique solvability of linear equations determined by monotone decomposable operators
}

\author{
Andrei Rontó
}




\title{
ON THE UNIQUE SOLVABILITY OF LINEAR EQUATIONS DETERMINED BY MONOTONE DECOMPOSABLE OPERATORS
}

\author{
ANDREI RONTÓ \\ [Received: January 5, 2004]

\begin{abstract}
Conditions sufficient for the unique solvability of the linear equation$$
u=A_{1} u-A_{2} u+z
$$

for arbitrary $z$ are established, where $A_{i}: X \rightarrow X, i=1,2$, are compact linear operators preserving a wedge in the Banach space $X$. The conditions obtained have the form of one-sided order relations satisfied by the values of $A_{1}$ and $A_{2}$ at certain "strongly positive," in a sense, elements of the space.
\end{abstract}

Mathematics Subject Classification: 46B40, 34K30, 46N20

Keywords: Wedge, operator preserving a wedge, spectral radius, test element

\section{InTroduction}

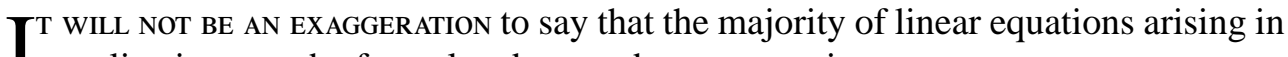
applications can be formulated as an abstract equation

$$
u=A u+z
$$

where $A: X \rightarrow X$ is a bounded linear operator in a suitably chosen Banach space $X$. Such an equation can often be represented in the form

$$
u=A_{1} u-A_{2} u+z
$$

where $A_{1}$ and $A_{2}$ are monotone in some or another sense. A natural definition of monotonicity is based upon the notion of a wedge (or, alternatively, a linear semigroup) in a Banach space, which dates back to the works of M. Krein [5]; this approach will be used below. Thus, we consider here equation (1.2) on the assumption that the operators $A_{1}$ and $A_{2}$ preserve a certain wedge in the given space $X$ (see Section 2 for the basic definitions).

The standard condition

$$
r\left(A_{1}-A_{2}\right)<1
$$

Supported in part by the NATO Science Fellowships Programme for the Czech Republic, Grant 7/2003. 
for the spectral radius $r\left(A_{1}-A_{2}\right)$ of the operator $A_{1}-A_{2}$, as is well-known, guarantees the existence and boundedness of the inverse operator

$$
\left(\mathbf{1}_{X}-A_{1}+A_{2}\right)^{-1}: X \rightarrow X
$$

and, hence, the existence of a unique solution of equation (1.2) for an arbitrary $z$ from $X$ and its continuous dependence on $z$. This proposition is, in a sense, unimprovable, but has the disadvantage that the fulfilment of inequality (1.3) can be verified directly only in exceptional cases. It is, therefore, desirable to have some statements guaranteeing estimate (1.3) on the base of information which can be comparatively easily extracted from the definition of the operators considered. For positive, in a sense, linear operators $A: X \rightarrow X$, namely, for those preserving a suitable cone in $X$, there is a series of efficient theorems (see, e. g., $[8,4,3]$ ) allowing one to derive bounds for the spectral radius of $A$ from relations involving the values of $A$ at a single non-zero element of $X$ (such an element, of course, should be suitably chosen, in accordance with the conditions assumed). This group of theorems is sometimes referred to as Krein's method of test elements.

The theorems mentioned above, however, have a certain limitation which restricts the field of their applicability. Namely, it is assumed that the operator under consideration preserves a cone satisfying some additional conditions (in particular, possessing the property of normality). Such conditions, despite their considerable generality, are however not satisfied in many important cases where the "positivity" is lacking. To overcome this difficulty, an approach different from those used in the works cited was suggested in $[7,6]$. Some of the results obtained therein are used in the present note (see Section 2.3).

Here, we are interested in conditions sufficient for the unique solvability of equation (1.2) with $A_{1}$ and $A_{2}$ preserving a wedge $P$ in the space $X$, i. e., such that

$$
A_{1}(P) \cup A_{2}(P) \subset P .
$$

The difference between the approach of [6] and that of the present note is that here conditions are imposed separately on the "positive" and "negative" parts of the equation, for which purpose a certain decomposition trick is applied (Section 3). The proofs of the results of Section 4 use the "positive" version of Theorem 4.1 from [6].

\section{Preliminaries}

Let $X$ be a Banach space over the field $\mathbb{R}$ and $P$ be a wedge in $X$. Recall that a closed set $P \subset X$ is called a wedge [3] (or a linear semigroup [5]) if

$$
\alpha_{1} P+\alpha_{2} P \subset P
$$

for all $\left\{\alpha_{1}, \alpha_{2}\right\} \subset(0,+\infty)$, where, by definition,

$$
\alpha_{1} \Omega+\alpha_{2} \Omega:=\left\{\alpha_{1} u_{1}+\alpha_{2} u_{2} \mid\left\{u_{1}, u_{2}\right\} \subset \Omega\right\} .
$$

for an arbitrary set $\Omega \subset X$ and all real $\alpha_{1}$ and $\alpha_{2}$. 
The linear manifold

$$
\text { bl } P:=P \cap(-P)
$$

is referred to as the blade [3] of the wedge $P$. A wedge having the trivial blade is called a cone [5].

2.1. Preorderings. Every wedge $P$ allows one to introduce a natural preordering " $\geqq_{P}$ " in the space $X$ by putting

$$
u_{1} \geqq_{P} u_{2} \text { if, and only if } u_{1}-u_{2} \in P .
$$

We shall also write $u_{1} \varliminf_{P} u_{2}$ if, and only if $u_{2} \geqq_{P} u_{1}$. Note that the relations $u_{1} \geqq_{P} u_{2}$ and $u_{2} \geqq_{P} u_{1}$ satisfied simultaneously, generally speaking, do not imply the equality $u_{1}=u_{2}$, unless bl $P=\{0\}$.

A wedge $P$ is said to be solid [5] if its interior is non-empty. In this case, following [5], we define the relation " $\gg_{P}$ " by setting

$$
u_{1} \gg_{P} u_{2} \text { if, and only if } u_{1}-u_{2} \text { is an interior element of } P \text {. }
$$

An element $u$ from $X$ is said to be $f$-measurable $[2,3]$ with respect to $P$ if there exists a constant $\beta \in[0,+\infty)$ such that

$$
-\beta f \leqq_{P} u \leqq_{P} \beta f .
$$

Here, $f$ is a certain fixed non-zero element from $X$.

Proposition 2.1. The set

$$
X_{P}(f):=\{u \in X \mid u \text { is } f \text {-measurable with respect to } P\}
$$

is non-empty if, and only if $f \geqq_{P} 0$. Furthermore, for an arbitrary element $f$ from $P$, the set $X_{P}(f)$ is a linear manifold in $X$.

This statement is readily obtained directly from the definitions formulated above.

2.2. Strict inequalities. Let $H$ be a certain linear manifold in $X$ (not necessarily a closed one).

Definition 2.2 ([6]). For $\left\{f_{1}, f_{2}\right\} \subset X$, we write $f_{1} \prec_{P ; H} f_{2}$ if, and only if the inclusion

$$
X_{P}\left(f_{2}-f_{1}\right) \supset H
$$

is true.

Similarly, we write $f_{1}>_{P ; H} f_{2}$ if, and only if $f_{2} \prec_{P ; H} f_{1}$. Note that, by virtue of Proposition 2.1, $X_{P}(f)$ is a linear manifold for all $f$ from $P$.

Definition 2.3 ([6]). For $\left\{f_{1}, f_{2}\right\} \subset X$, we write $f_{1} \prec_{P} f_{2}$ if, and only if the equality

$$
X_{P}\left(f_{2}-f_{1}\right)=X
$$

is true.

Analogously, we write $f_{1}>_{P} f_{2}$ if, and only if $f_{2} \prec_{P} f_{1}$. 
Example 2.4. If, for instance, $X=C([a, b], \mathbb{R}),{ }^{*}$ the wedge $P$ is defined by the formula

$$
P=\{u \in C([a, b], \mathbb{R}) \mid u(t) \geq 0 \text { for all } t \text { from }[a, b]\},
$$

and $f$ is the superlinear function given by the equality

$$
f(t)=|t-\tau|^{\alpha}, \quad t \in[a, b]
$$

with some $\tau \in[a, b]$ and $\alpha \in[1,+\infty)$, then, as is easy to see, the corresponding set (2.3) is described by the formula

$$
X_{P}(f)=\left\{u \in C([a, b], \mathbb{R})\left|\sup _{t \in[a, b]}\right|(t-\tau)^{-\alpha} u(t) \mid<+\infty\right\} .
$$

This set, clearly, forms a proper linear submanifold in $C([a, b], \mathbb{R})$. Every function belonging to the set mentioned, in particular, vanishes at the point $\tau$.

The relations " $<_{P ; H}$ " and " $<_{P}$ " provide us a kind of "strict inequalities" whose properties resemble, to some extent, those of the componentwise sign " $>$ " in $\mathbb{R}^{n}$, $n \in \mathbb{N}$. For example, it is not difficult to verify that

$$
f>_{P} 0 \text { implies that } f \geqq_{P} 0
$$

and

$$
f \geqq_{P} g \text { and } g>_{P} 0 \text { imply } f>_{P} 0 .
$$

An efficient condition sufficient for the relation

$$
f>_{P} 0
$$

to be fulfilled is provided by the following lemma.

Lemma 2.5. If $P$ is a solid wedge in $X$ and an element $f$ is such that $f \gg_{P} 0$, then $f$ satisfies relation (2.4).

Proof. A statement equivalent to the equality $X_{P}(f)=X$ for $f$ lying in the interior of $P$ is well-known, e. g., from [5].

2.3. Spectrum of monotone decomposable operators. Let us first introduce the following standard

Definition 2.6. An operator $A: X \rightarrow X$ is said to preserve the wedge $P$ if $A(P) \subset P$.

The following statement has recently been obtained in [6].

Theorem 2.7 ([6]). Let $P$ be a wedge in $X$ and $A_{1}: X \rightarrow X, A_{2}: X \rightarrow X$ be completely continuous linear operators preserving $P$, satisfying the condition

$$
\text { bl } P \subset \operatorname{ker}\left(A_{1}-A_{2}\right) \text {, }
$$

and, moreover, such that

$$
\operatorname{im}\left(A_{1}-A_{2}\right) \subset H,
$$

${ }^{*}$ The symbol $C([a, b], \mathbb{R})$ here stands for the Banach space of all continuous functions $u:[a, b] \rightarrow$ $\mathbb{R}$ equipped with the usual norm $\|u\|:=\max _{t \in[a, b]}|u(t)|$. 
where $H$ is a certain given linear manifold from $X$. If, in addition, the inequality

$$
\alpha f \geqq_{P} A_{1} f+A_{2} f
$$

holds with some constant $\alpha \in[0,+\infty)$ and element $f \in P \backslash\{0\}$ satisfying the condition

$$
f>_{P ; H} 0,
$$

then the spectral radius $r\left(A_{1}-A_{2}\right)$ of the operator $A_{1}-A_{2}$ admits the estimate

$$
r\left(A_{1}-A_{2}\right) \leq \alpha .
$$

Theorem 2.7 implies, in particular, the following corollaries.

Corollary 2.8. Let $P$ be a solid wedge in $X$ and $A_{1}: X \rightarrow X, A_{2}: X \rightarrow X$ be completely continuous linear operators preserving $P$, satisfying condition (2.5), and such that inequality (2.6) is true with some constant $\alpha \in[0,+\infty)$ and element $f \in$ $P \backslash\{0\}$ possessing the property

$$
f \gg_{P} 0 \text {. }
$$

Then the spectral radius $r\left(A_{1}-A_{2}\right)$ of the operator $A_{1}-A_{2}$ admits estimate (2.8). Proof. In view of Lemma 2.5, it suffices to apply Theorem 2.7 with $H=X$.

Corollary 2.9. Let $P$ be a wedge in $X$ and $A: X \rightarrow X$ be a completely continuous linear operator preserving $P$ and satisfying the conditions

$$
\text { bl } P \subset \operatorname{ker} A
$$

and

$$
\operatorname{im} A \subset H,
$$

where $H$ is a certain linear manifold in $X$. If, moreover, there exists an $\alpha \in[0,+\infty)$ and a certain element $f$ satisfying condition (2.7) and the relation

$$
\alpha f \geqq_{P} A f,
$$

then the spectral radius $r(A)$ of the operator $A$ admits the estimate

$$
r(A) \leq \alpha .
$$

Proof. Application of Theorem 2.7 with $A_{1}=A$ and $A_{2}=0$.

Corollary 2.10 ([7]). Assume that $P$ is a solid wedge in $X$ and $A: X \rightarrow X$ is a completely continuous linear operator preserving $P$, satisfying condition (2.10) and, moreover, such that relation (2.12) is true with certain $\alpha \in[0,+\infty)$ and an $f \in P$ possessing property (2.9). Then the spectral radius of A admits estimate (2.13).

Proof. It is sufficient to apply Corollary 2.8 with $A_{1}=A$ and $A_{2}=0$.

\section{A deCOMposition techniQue}

Let $X$ be a Banach space, $P$ be a wedge in $X$, and $A_{1}, A_{2}: X \rightarrow X$ be bounded linear operators such that inclusion (1.5) is true. 
3.1. The decomposition lemma. The following simple lemma allows one to rewrite equation (1.2) in a different form which may sometimes prove more convenient due to the presence of assumption (1.5).

Lemma 3.1. If a pair $\left(u_{1}, u_{2}\right) \subset X \times X$ is a solution of the system

$$
\begin{aligned}
& u_{1}=A_{1} u_{1}+A_{2} u_{2}+z_{1}, \\
& u_{2}=A_{2} u_{1}+A_{1} u_{2}+z_{2}
\end{aligned}
$$

with some $\left(z_{1}, z_{2}\right) \subset X \times X$, then the element

$$
u=u_{1}-u_{2}
$$

is a solution of equation (1.2) with $z:=z_{1}-z_{2}$. Conversely, if $u \in X$ is a solution of equation (1.2) with a certain $z \in X$, then there exist pairs $\left(u_{1}, u_{2}\right) \in X^{2}$ and $\left(z_{1}, z_{2}\right) \in X^{2}$ such that equalities (3.3), (3.1), and (3.2) are satisfied.

Proof. If $\left(u_{1}, u_{2}\right)$ satisfies (3.1), (3.2), then, obviously,

$$
u_{1}-u_{2}=\left(A_{1}-A_{2}\right) u_{1}+\left(A_{2}-A_{1}\right) u_{2}+z_{1}-z_{2}
$$

and, therefore, equality (1.2) is satisfied with $u:=u_{1}-u_{2}$ and $z:=z_{1}-z_{2}$.

Conversely, assume that $u$ is a solution of equation (1.2). Let $u_{1}$ and $u_{2}$ be some elements of $X$ such that equality (3.3) is true. Then (3.1) and (3.2) are satisfied with $z_{1}:=z+u_{2}-A_{1} u_{2}-A_{2} u_{1}$ and $z_{2}:=u_{2}-A_{1} u_{2}-A_{2} u_{1}$.

Despite its extreme simplicity, Lemma 3.1 nevertheless allows one to obtain meaningful results on the unique solvability of equation (1.2).

3.2. Unique solvability of system (3.1), (3.2). The following propositions provide conditions sufficient for the unique solvability of system (3.1), (3.2) with arbitrary values of $z_{1}$ and $z_{2}$.

Proposition 3.2. Let $A_{1}$ and $A_{2}$ be bounded linear operators satisfying the conditions

$$
r\left(A_{1}\right)<1
$$

and

$$
r\left(A_{1}+A_{2}\left(\mathbf{1}_{X}-A_{1}\right)^{-1} A_{2}\right)<1 .
$$

Then system (3.1), (3.2) is uniquely solvable for arbitrary $\left\{z_{1}, z_{2}\right\} \subset X$, and its solution $\left(u_{1}, u_{2}\right)$ can be represented by the formula

$$
\begin{aligned}
& u_{1}=\hat{A}_{1} z_{1}+\hat{A}_{1} A_{2}\left(\mathbf{1}_{X}-A_{1}-A_{2} \hat{A}_{1} A_{2}\right)^{-1}\left[z_{2}+A_{2} \hat{A}_{1} z_{1}\right], \\
& u_{2}=\left(\mathbf{1}_{X}-A_{1}-A_{2} \hat{A}_{1} A_{2}\right)^{-1}\left[z_{2}+A_{2} \hat{A}_{1} z_{1}\right],
\end{aligned}
$$

where

$$
\hat{A}_{1}:=\left(\mathbf{1}_{X}-A_{1}\right)^{-1} .
$$

If, moreover, $A_{1}$ and $A_{2}$ preserve the wedge $P$, then the solution of system (3.1), (3.2) satisfies the condition $\left(u_{1}, u_{2}\right) \in P^{2}$ whenever $\left(z_{1}, z_{2}\right) \in P^{2}$. 
Proof. It follows from (3.4) that operator (3.8) is well-defined and, hence, (3.1) is equivalent to the equation

$$
u_{1}=\hat{A}_{1}\left[z_{1}+A_{2} u_{2}\right]
$$

Substituting (3.9) into (3.2), we obtain

$$
u_{2}=A_{2} \hat{A}_{1}\left[z_{1}+A_{2} u_{2}\right]+A_{1} u_{2}+z_{2}
$$

or, which is the same,

$$
u_{2}=\left[A_{1}+A_{2} \hat{A}_{1} A_{2}\right] u_{2}+z_{2}+A_{2} \hat{A}_{1} z_{1} .
$$

Condition (3.5) guarantees the existence of the inverse operator

$$
\left(\mathbf{1}_{X}-A_{1}-A_{2} \hat{A}_{1} A_{2}\right)^{-1}
$$

and, therefore, equation (3.10) has a unique solution $u_{2}$ given by formula (3.7). Inserting (3.7) into (3.1) and taking (3.9) into account, we arrive at (3.6).

By virtue of (3.4) and (3.5), the bounded linear operators (3.8) and (3.11) are representable by the corresponding Neumann series

$$
\hat{A}_{1}=\mathbf{1}_{X}+A_{1}+A_{1}^{2}+\ldots
$$

and

$$
\left(\mathbf{1}_{X}-A_{1}-A_{2} \hat{A}_{1} A_{2}\right)^{-1}=\mathbf{1}_{X}+A_{1}+A_{2} \hat{A}_{1} A_{2}+\left(A_{1}+A_{2} \hat{A}_{1} A_{2}\right)^{2}+\ldots,
$$

whence it follows that operators (3.8) and (3.11) preserve the wedge $P$ provided that $A_{1}$ and $A_{1}$ do so. This fact, in view of representation (3.6), (3.7) for the unique solution $\left(u_{1}, u_{2}\right)$ of system (3.1), (3.2), implies that, under assumption (1.5), the solution mentioned satisfies the relations $u_{1} \geqq_{P} 0$ and $u_{2} \geqq_{P} 0$ whenever $z_{1} \geqq_{P} 0$ and $z_{2} \geqq_{P} 0$.

Proposition 3.3. Let $A_{1}: X \rightarrow X$ and $A_{2}: X \rightarrow X$ be bounded linear operators such that inequality (3.4) is true and, moreover,

$$
r\left(\left(\mathbf{1}_{X}-A_{1}\right)^{-1} A_{2}\right)<1 .
$$

Then system (3.1), (3.2) is uniquely solvable for arbitrary $\left(z_{1}, z_{2}\right) \in X^{2}$, and its solution $\left(u_{1}, u_{2}\right)$ admits representation in the form

$$
\begin{aligned}
& u_{1}=\hat{A}_{1} z_{1}+\hat{A}_{1} A_{2}\left(\mathbf{1}_{X}-A_{2}^{2} \hat{A}_{1}^{2}\right)^{-1} \hat{A}_{1}\left[z_{2}+A_{2} \hat{A}_{1} z_{1}\right], \\
& u_{2}=\left(\mathbf{1}_{X}-A_{2}^{2} \hat{A}_{1}^{2}\right)^{-1} \hat{A}_{1}\left[z_{2}+A_{2} \hat{A}_{1} z_{1}\right] .
\end{aligned}
$$

If, moreover, $A_{1}$ and $A_{2}$ satisfy condition (1.5), then $u_{1} \geqq_{P} 0$ and $u_{2} \geqq_{P} 0$ whenever $z_{1} \geqq_{P} 0$ and $z_{2} \geqq_{P} 0$.

Proof. By virtue of (3.4), operator (3.8) is well-defined and, hence (3.2) can be rewritten as

$$
u_{2}=\hat{A}_{1}\left[z_{2}+A_{2} u_{1}\right]
$$


whereas equation (3.1) can be brought to form (3.9). Substituting (3.9) into (3.16), we get

$$
\begin{aligned}
u_{2} & =\hat{A}_{1}\left[z_{2}+A_{2} \hat{A}_{1} z_{1}+A_{2} \hat{A}_{1} A_{2} u_{2}\right] \\
& =\left(\hat{A}_{1} A_{2}\right)^{2} u_{2}+\hat{A}_{1}\left(z_{2}+A_{2} \hat{A}_{1} z_{1}\right) \\
& =A_{2}^{2} \hat{A}_{1}^{2} u_{2}+\hat{A}_{1}\left(z_{2}+A_{2} \hat{A}_{1} z_{1}\right) .
\end{aligned}
$$

For any bounded linear operator $B: X \rightarrow X$, the inequality $r\left(B^{2}\right)<1$ holds if, and only if $r(B)<1$. Therefore, in view of (3.8), assumption (3.13) guarantees the existence of a bounded inverse operator

$$
\left(\mathbf{1}_{X}-A_{2}^{2} \hat{A}_{1}^{2}\right)^{-1}
$$

whence it follows that the unique element $u_{2}$ satisfying relation (3.17) is given by equality (3.15). Substituting (3.15) into (3.9), we obtain (3.14).

Finally, by virtue of (3.4) and (3.13), it follows from formulae (3.14) and (3.15) that, under condition (1.5), the inclusion $\left(z_{1}, z_{2}\right) \in P^{2}$ implies the property $\left(u_{1}, u_{2}\right) \in$ $P^{2}$ for the unique solution $\left(u_{1}, u_{2}\right)$ of system (3.1), (3.2).

Propositions 3.2 and 3.3 lead one to the following statement.

Proposition 3.4. Let $A_{1}: X \rightarrow X$ and $A_{2}: X \rightarrow X$ be bounded linear operators satisfying condition (3.4) and one of relations (3.5) and (3.13). Then 1 is a regular value for the operator $A_{1}-A_{2}$.

Proof. According to Propositions 3.2 and 3.3, each of the pairs of conditions (3.4), (3.5) and (3.4), (3.13) guarantees the unique solvability of system (3.1), (3.2) for all $\left(z_{1}, z_{2}\right) \in X^{2}$. Formulae (3.6), (3.7) and (3.14), (3.15), which represent the unique solution of this system in the respective cases, imply, in particular, that the solution mentioned depends continuously on $\left(z_{1}, z_{2}\right)$. Therefore, in view of Lemma 3.1, the conditions indicated guarantee that equation (1.2) has a unique solution for an arbitrary $z \in X$, and the dependence of this solution on $z$ is continuous. Thus, there exists a bounded inverse operator (1.4), i. e., 1 is a regular value for $A_{1}-A_{2}$.

\section{UNIQUe SOLVABILITY OF EQUATION (1.2)}

Proposition 4.1. Let $P$ be a wedge in the space $X$ and $A_{1}$ and $A_{2}$ be completely continuous linear operators in $X$ preserving $P$, satisfying the condition

$$
\text { bl } P \subset \operatorname{ker} A_{1} \cap \operatorname{ker} A_{2} \text {, }
$$

and such that ${ }^{\dagger}$

$$
\begin{gathered}
\operatorname{im} A_{1} \subset H_{1}, \\
\operatorname{im} A_{1}+\operatorname{im} A_{2} \subset H_{2},
\end{gathered}
$$

${ }^{\dagger}$ Recall that we use notation (2.1). Inclusion (4.3) thus means that $A_{1} u_{1}+A_{2} u_{2} \in H_{2}$ for arbitrary $u_{1}$ and $u_{2}$ from $X$. 
where $H_{1}$ and $H_{2}$ are certain linear manifolds in $X$. Assume also that the relations

$$
A_{1} f_{1} \leqq_{P} \alpha_{1} f_{1}
$$

and

$$
A_{1} f_{2}+A_{2}\left(\mathbf{1}_{X}-A_{1}\right)^{-1} A_{2} f_{2} \leqq_{P} \alpha_{2} f_{2}
$$

are true with some $\left\{\alpha_{1}, \alpha_{2}\right\} \subset[0,1)$ and $\left\{f_{1}, f_{2}\right\} \subset P$ such that

$$
f_{1}>_{P ; H_{1}} 0
$$

and

$$
f_{2}>_{P ; H_{2}} 0 \text {. }
$$

Then inequality (1.3) is true.

Remark 4.2. As follows from the proof below, the existence of the inverse operator

$$
\left(\mathbf{1}_{X}-A_{1}\right)^{-1}: X \rightarrow X
$$

involved in inequality (4.5) is guaranteed by the rest of the conditions assumed and, thus, relation (4.5) makes sense.

Proof of Proposition 4.1. By virtue of Corollary 2.9, assumptions (4.1), (4.2), (4.4), and (4.6) guarantee that operator (4.8) is well-defined.

It is obvious that the inclusion

$$
\operatorname{im}\left[A_{1}+A_{2}\left(\mathbf{1}_{X}-A_{1}\right)^{-1} A_{2}\right] \subset \operatorname{im} A_{1}+\operatorname{im} A_{2}
$$

is true and, hence, in view of (4.3), the operator

$$
A:=A_{1}+A_{2}\left(\mathbf{1}_{X}-A_{1}\right)^{-1} A_{2}
$$

satisfies the condition im $A \subset H_{2}$. Since conditions (4.1), (4.5), and (4.7) are assumed to be satisfied, we see that Corollary 2.9 can be applied with $f:=f_{2}, \alpha:=\alpha_{2}$, and $A$ given by formula (4.9). Application of Corollary 2.9 implies inequality (3.5) for the spectral radius of operator (4.9), whence, by Proposition 3.2, it follows that system (3.1), (3.2) is uniquely solvable for arbitrary $z_{1}$ and $z_{2}$. Formulae (3.6) and (3.7) representing the unique solution of system (3.1), (3.2) show that its dependence on $\left(z_{1}, z_{2}\right)$ is continuous and, hence, taking Lemma 2.5 into account, we conclude that the spectral radius of the operator $A_{1}-A_{2}$ satisfies the required inequality (1.3).

To obtain more constructive conditions sufficient for the unique solvability of equation (1.2), namely, Theorems 4.4 and 4.6 below, we need the following simple supplement to Corollary 2.9.

Lemma 4.3. Let $P$ be wedge in $X$ and $A: X \rightarrow X$ be a completely continuous linear operator preserving $P$, satisfying conditions (2.10) and (2.11) with a certain linear manifold $H \subset X$ and, moreover, such that there exists an element $f \in P$ for which relations (2.7) and (2.12) are true with a certain constant $\alpha, 0 \leq \alpha<1$. 
Then the inverse operator

$$
\left(\mathbf{1}_{X}-A\right)^{-1}: X \rightarrow X
$$

exists, is bounded, preserves the wedge $P$, and, furthermore, the relation

$$
\left(\mathbf{1}_{X}-A\right)^{-1} f \leqq_{P} \frac{1}{1-\alpha} f
$$

is true.

Proof. Corollary 2.9 guarantees that operator (4.10) is well-defined and bounded. By assumption, $A$ preserves $P$ and, hence, inequality (2.12) implies that $A^{i} f \leqq_{P} \alpha^{i} f$ for all $i=0,1,2, \ldots$. Using now the Neumann series representation of operator (4.10), we arrive at the relation

$$
\left(\mathbf{1}_{X}-A\right)^{-1} f=\sum_{i=0}^{+\infty} A^{i} f \leqq_{P} \sum_{i=0}^{+\infty} \alpha^{i} f,
$$

which, clearly, yields (4.11).

Theorem 4.4. Let $A_{1}: X \rightarrow X, A_{2}: X \rightarrow X$ be completely continuous linear operators preserving a wedge $P$ in $X$ and satisfying condition (4.1). Assume the existence of an element $f \in P$ satisfying condition (2.4), possessing the property

$$
A_{1}^{i} A_{2} f \leqq_{P} A_{2} A_{1}^{i} f \quad \text { for all } i=0,1,2, \ldots,
$$

and such that the relations

$$
\begin{gathered}
A_{1} f \varliminf_{P} \alpha f, \\
A_{2}^{2} f \varliminf_{P} \gamma(1-\alpha) f
\end{gathered}
$$

are true with certain constants $\alpha \in[0,1)$ and $\gamma \in[0,1-\alpha)$.

Then 1 is a regular value of the operator $A_{1}-A_{2}$.

Proof. Let us show that the assumptions of Proposition 4.1 are satisfied with $f_{1}=f$, $f_{2}=f, \alpha_{1}=\alpha, \alpha_{2}=\gamma+\alpha, H_{1}=X$, and $H_{2}=X$.

Indeed, (4.13) is nothing but (4.4) with $\alpha_{1}$ replaced by $\alpha$. Condition (4.5) with $f_{2}=f$ has the form

$$
A_{1} f+A_{2}\left(\mathbf{1}_{X}-A_{1}\right)^{-1} A_{2} f \leqq_{P} \alpha_{2} f .
$$

Considering the Neumann series (3.12) for operator (3.8), we get

$$
\left(\mathbf{1}_{X}-A_{1}\right)^{-1} A_{2} f=\sum_{i=0}^{+\infty} A_{1}^{i} A_{2} f
$$

whence, by taking assumptions (4.12), (4.13), and (1.5) into account and applying Lemma 4.3 with $H=X$ and $A=A_{1}$, we obtain

$$
\left(\mathbf{1}_{X}-A_{1}\right)^{-1} A_{2} f \leqq_{P} A_{2} \sum_{i=0}^{+\infty} A_{1}^{i} f=A_{2}\left(\mathbf{1}_{X}-A_{1}\right)^{-1} f \leqq_{P} \frac{1}{1-\alpha} A_{2} f .
$$


Therefore, for relation (4.15) to be true, it would suffice that

$$
A_{1} f+\frac{1}{1-\alpha} A_{2}^{2} f \leqq_{P} \alpha_{2} f .
$$

In view of (4.13), relation (4.16) is satisfied whenever

$$
\alpha f+\frac{1}{1-\alpha} A_{2}^{2} f \leqq P \alpha_{2} f
$$

i. e., if

$$
A_{2}^{2} f \leqq_{P}(1-\alpha)\left(\alpha_{2}-\alpha\right) f .
$$

Condition (4.17) coincides with (4.14) if we put $\alpha_{2}:=\gamma+\alpha$. Note that this value of $\alpha_{2}$ is such that $0 \leq \alpha_{2}<1$ because, by assumption, $0 \leq \gamma<1-\alpha$. Finally, according to Definition 2.3, condition (2.4) is a particular case of (4.6) with $f_{1}=f$ and $H_{1}=X$, and it remains to refer to Proposition 4.1 to obtain the conclusion desired.

Remark 4.5. Condition (4.12) is satisfied for every $f \geqq_{P} 0$ if $A_{1}$ and $A_{2}$ are such that

$$
A_{1} A_{2} u \varliminf_{P} A_{2} A_{1} u \text { for all } u \in P .
$$

Another kind of conditions ensuring the unique solvability of equation (1.2) can be obtained by using Proposition 3.3.

Theorem 4.6. Let $A_{1}: X \rightarrow X, A_{2}: X \rightarrow X$ be completely continuous linear operators preserving a wedge $P \subset X$ and satisfying condition (4.1) and the relation

$$
\operatorname{im} A_{1} \subset H
$$

with a certain linear manifold $H \subset X$. Let, moreover, the relations

$$
\begin{gathered}
A_{1} A_{2} g \leqq_{P} \alpha A_{2} g, \\
A_{2}^{2} g \leqq_{P} \beta(1-\alpha) g
\end{gathered}
$$

hold, where $\{\alpha, \beta\} \subset[0,1)$ and $g \in P$ is an element having the following properties:

$$
g>_{P} 0
$$

and

$$
A_{2} g>_{P ; H} 0 .
$$

Then 1 is a regular value of the operator $A_{1}-A_{2}$.

Condition (4.18) implies, in particular, that the operator $\left[A_{1}, A_{2}\right]=A_{1} A_{2}-A_{2} A_{1}$ preserves the blade bl $P$ of the wedge $P$. In the case where $P$ is a cone (i. e., if bl $P=\{0\})$, a pair of operators $\left(A_{1}, A_{2}\right)$ satisfying (4.18) is sometimes referred to as semicommuting [1]. 
Proof. Conditions (4.19), (4.20), and (4.23) guarantee that the inverse operator (4.8) is well-defined and bounded (it suffices to apply Corollary 2.9 with $A:=A_{1}$ and $f:=A_{2} g$ ). Let us show that the rest of assumptions ensure that relation (3.13) holds.

Indeed, according to Corollary 2.9, it will suffice to show that the relation

$$
\left(\mathbf{1}_{X}-A_{1}\right)^{-1} A_{2} f \leqq_{P} \gamma f
$$

is true with some $\gamma \in[0,1)$ and $f$ satisfying (2.4). However, by virtue of Lemma 4.3 applied with $f=A_{2} g$, we have

$$
\left(\mathbf{1}_{X}-A_{1}\right)^{-1} A_{2} A_{2} g \leqq_{P} \frac{1}{1-\alpha} A_{2} g
$$

and, hence, (4.24) will be satisfied for $f=A_{2} g$ provided that

$$
\frac{1}{1-\alpha} A_{2}^{2} g \leqq_{P} \gamma A_{2} g \text {. }
$$

Note now that (4.21) is nothing but (4.25) with $\gamma:=\beta$.

Remark 4.7. To every statement appearing in Sections 3.2 and 4, a dual one corresponds, with the rôles of $A_{1}$ and $A_{2}$ interchanged.

\section{REFERENCES}

[1] Esajan, A. R. And SrožIdDinov, H. M.: The comparison of the spectral radii of semicommuting operators, Sibirsk. Mat. Zh., 12 (1971), 332-345.

[2] Krasnoselskit, M. A.: Positive Solutions of Operator Equations, Noordhoff, Groningen, 1964.

[3] Krasnoselskit, M. A., Lifshits, E. A., and Sobolev, A. V.: Positive Linear Systems. Method of Positive Operators, Heldermann, Berlin, 1989.

[4] Krasnoselskit, M. A., VainikKo, G. M., Zabreiko, P. P., Rutitskit, Ya. B., and Stetsenko, V. Ya.: Approximate Solution of Operator Equations, Noordhoff, Groningen, 1972.

[5] Krein, M. G. and Rutman, M. A.: Linear Operators Leaving Invariant a Cone in a Banach Space, No. 26 in Amer. Math. Soc. Transl., Amer. Math. Soc., R. I., 1950.

[6] RonTó, A.: Bounds for the spectrum of compact linear operators in a preordered Banach space, submitted.

[7] RonTó, A.: On substantial eigenvalues of linear operators leaving invariant a closed wedge, In: CDDE-2002 Proceedings, vol. 13 of Folia FSN Universitatis Masarykianae, Mathematica, Masaryk University, 2003, pp. 235-246.

[8] Zabreiko, P. P., Krasnoselskit, M. A., and Stetsenko, V. Y.: Estimates of the spectral radius of postive linear operators, Mat. Zametki, 1 (1967), 461-468.

\section{Author's Address}

\section{Andrei Rontó:}

Institute of Mathematics, National Academy of Sciences of Ukraine, 3 Tereschenkovskaya St., 01601 KIEv, UkRAINE

Current address: Mathematical Institute, Czech Academy of Sciences, Žižkova 22, CZ-61662 Brno,

Czech Republic

E-mail address: ronto@ipm.cz 